\title{
The use of contrast-enhanced, time-resolved magnetic resonance angiography in cerebrovascular pathology
}

\author{
*Jonathan A. Grossberg, MD, ${ }^{1}$ Brian M. Howard, MD, ${ }^{1,2}$ and Amit M. Saindane, MD2 \\ Departments of ${ }^{1}$ Neurosurgery and ${ }^{2}$ Radiology and Imaging Sciences, Emory University School of Medicine, Atlanta, Georgia
}

Digital subtraction angiography (DSA) has long been the imaging gold standard in the evaluation, treatment, and followup of cerebro- and spinovascular disorders. However, DSA has the disadvantages of invasiveness, contrast allergy or nephropathy, the impracticality of procedural preparation and recovery, and expense. Contrast-enhanced (CE), timeresolved (TR) magnetic resonance angiography (CE TR-MRA) is a sophisticated, relatively novel imaging modality that provides multiphasic contrast-enhanced visualization of the neurovasculature. Given the crucial role of angiography in all aspects of care for patients with complex neurovascular disorders, it is incumbent on those who care for these patients to understand the usefulness and pitfalls of novel imaging in this arena to ensure best practices, and to deliver cutting edge care to these patients in a way that minimizes cost, but does not compromise quality. CE TR-MRA has the potential to play an expanded role in the workup and follow-up across the spectrum of neurovascular disease, and this review is aimed to help neurosurgeons better understand how CE TR-MRA can be used to better manage patients in this cohort. https://thejns.org/doi/abs/10.3171/2019.9.FOCUS19627

KEYWORDS time-resolved MRA; aneurysm; arteriovenous malformation; AVM; arteriovenous fistula; AVF

$\mathrm{W}$ HILE digital subtraction angiography (DSA) remains the gold standard for imaging for cerebroand spinovascular pathologies, noninvasive modalities have an ever-expanding role in the management of patients with these pathologies. While most clinicians are familiar with CT angiography (CTA) and time-of-flight (TOF) MR angiography (MRA), less is commonly known about more advanced MRA techniques such as contrastenhanced (CE), time-resolved (TR) MRA (CE TR-MRA).

TR-MRA exploits various approaches to acceleration of image acquisition including parallel imaging, undersampling of k-space, and view sharing, to generate multiphasic angiographic images during the administration of intravenous gadolinium contrast agent, resulting in temporal resolution of 2-6 seconds at submillimeter isotropic spatial resolution. ${ }^{11,12}$ While DSA provides temporal resolution on the orders of fractions of a second, CE TR-MRA provides a noninvasive hemodynamic assessment of lesion vascularity, including early versus delayed vessel filling and venous drainage, which makes it useful in the radio- graphic evaluation of a number of cerebro- and spinovascular pathologies.

\section{Arteriovenous Malformations}

Arteriovenous malformations (AVMs) are congenital lesions in which blood is shunted from enlarged arteries to dilated veins via a nidus of disorganized vessels with no intervening brain. The natural history and hemorrhage rates of AVMs depend on various features of their angioarchitecture, such as deep drainage, venous outlet stenosis, and presence of aneurysms. ${ }^{8}$ While DSA remains the reference standard for AVM imaging, noninvasive imaging such as CE TR-MRA is an alternative and accurate way to screen for and follow AVMs. Furthermore, many patients undergo AVM treatment, whether surgically, endovascularly, via stereotactic radiosurgery, or in some combination. The workup, treatment, and follow-up of these patients requires numerous imaging studies and maximizing noninvasive imaging where possible is attractive to these

ABBREVIATIONS AVM = arteriovenous malformation; $\mathrm{CDAVF}=$ cranial DAVF; $\mathrm{CE}=$ contrast enhanced; $\mathrm{CTA}=\mathrm{CT}$ angiography; DAVF = dural arteriovenous fistula; DSA = digital subtraction angiography; MIP = maximum intensity projection; MRA = MR angiography; NPV = negative predictive value; PPV = positive predictive value; $\mathrm{SDAVF}=$ spinal DAVF; SM = Spetzler-Martin; TOF = time-of-flight; TR = time-resolved.

SUBMITTED August 1, 2019. ACCEPTED September 5, 2019.

INCLUDE WHEN CITING DOI: 10.3171/2019.9.FOCUS19627.

* J.A.G. and B.M.H. contributed equally to this work. 
patients. Finally, in the current era after the controversial ARUBA trial (A Randomized Trial of Unruptured Brain Arteriovenous Malformations), ${ }^{21}$ the push to observe AVMs is increasing, which adds importance to the use of accurate noninvasive imaging. A number of studies have evaluated the usefulness of CE TR-MRA in the evaluation of AVMs.

The first question to answer when comparing CE TRMRA to DSA in the evaluation of AVMs is: is the diagnostic accuracy of these techniques acceptably equivalent? Machet et al. compared CE TR-MRA to DSA in 19 cases, and found that MRA was able to diagnose the AVM in 17 cases $(89.5 \%) .{ }^{17}$ In both cases of MRA failure, the nidus was smaller than $1 \mathrm{~cm}$ in size and an overlying hematoma was postulated to have hidden the nidus. Similarly, Cuong et al. found a $100 \%$ correlation between CE TR-MRA and DSA in 14 AVMs. ${ }^{7}$

While accuracy in detection is important, the evaluation of angioarchitecture is crucially important in planning treatment. A number of studies have evaluated the efficacy of CE TR-MRA in this arena. Machet et al. found good agreement between CE TR-MRA and DSA in evaluating nidus size and venous drainage $(\kappa=0.75,0.77)$, and moderate agreement $(\kappa=0.44)$ for arterial feeders. ${ }^{17}$ Another important aspect in the evaluation of AVMs is the Spetzler-Martin (SM) scale. As most neuroradiologists and neurosurgeons know, the morbidity and mortality of AVM treatment has been linked to SM grade. ${ }^{28}$ Oleaga et al. compared the imaging of 12 AVMs and found $100 \%$ concordance between CE TR-MRA and DSA in terms of SM grade. ${ }^{23}$ These results are correlated by other studies that have found concordance rates of $93 \%-100 \%$ between CE TR-MRA and DSA in AVM cases.7,13,14

Another potentially useful application of CE TR-MRA is in surveillance imaging after treatment. Soize et al. compared CE TR-MRA follow-up imaging with DSA in 37 patients treated by surgery, embolization, or radiosurgery, or a combination. They found concordance of the imaging modalities in 33 (89\%) of 37 cases. The 4 cases in which MRA did not detect a residual nidus were all small and previously embolized. ${ }^{27}$ Similarly, Hadizadeh et al. found $100 \%$ concordance in postsurgical CE TR-MRA and DSA, although it should be noted that in only 1 case was there a residual nidus after resection. ${ }^{13}$

The one potential limitation in treatment follow-up is susceptibility artifact from liquid embolic agents or surgical AVM clips that can limit the evaluation of the surrounding parenchyma.

In our clinical practice, we have begun to use CE TRMRA to evaluate suspected AVMs prior to DSA and as a first-line screen for symptoms after treatment. At this point, however, DSA still remains our main test to diagnose new AVMs and evaluate AVMs after treatment.

\section{Cranial Dural Arteriovenous Fistulas}

Cranial dural arteriovenous fistulas (cDAVFs) are acquired, high-flow shunting lesions between dural arteries and a dural venous sinus, dural lakes, and/or leptomeningeal veins without an intervening nidus. These lesions can range from benign-causing minimal symptoms, such as headache or pulsatile tinnitus - to lesions with a high risk of hemorrhage. In general, hemorrhage risk is associated with retrograde, leptomeningeal drainage as detailed in the Borden and Cognard classification schemes., ${ }^{4,6}$ Classically, DSA has been used to diagnose and follow these lesions, but more recently advanced MRA imaging has been utilized as an alternative.

In the past, given the potentially high risk of hemorrhage associated with DAVFs, patients with even mild symptoms, such as pulsatile tinnitus, were subjected to the gold-standard test of a full DS angiogram, despite its invasiveness and potential risk. As an alternative to that practice, studies have compared the accuracy of CE TRMRA with DSA in the diagnosis of cDAVFs. Meckel et al. evaluated 18 patients with treated $(n=9)$ and untreated $(n=9)$ cDAVFs and found $100 \%$ correlation between CE TR-MRA and DSA in terms of fistula identification. Cognard grading of the DAVFs, however, was not as accurate, with correlation in $77 \%$ and $85 \%$ of each reviewer's cases. ${ }^{20}$ Similarly, Farb et al. reviewed 42 cases of suspected fistulas and found that in 39 (93\%) of 42 cases, all 3 readers unanimously correlated CE TR-MRA with gold-standard DSA in terms of the presence of the fistula and the grading. In the remaining 3 cases, only 1 of the 3 readers disagreed with the results of the DSA..$^{10}$ Figure 1 demonstrates an example of CE TR-MRA used to identify a Borden type I DAVF.

In terms of treatment planning, Ertl et al. evaluated 24 transverse sinus DAVFs with DSA and CE TR-MRA, and found that CE TR-MRA was better than DSA in evaluating venous drainage and sinus involvement. ${ }^{9}$ The authors postulated that, in some cases, hemodynamic effects of DSA can lead to venous sinus pseudoocclusion, and that CE TR-MRA does not suffer from the same hemodynamic effect. In addition, the authors describe how the MRA demonstrated the fistula drainage pattern in relationship to the rest of the intracranial compartment better than the DSA. Although the authors found a $100 \%$ correlation between CE TR-MRA and DSA in terms of DAVF grading, they did find that CE TR-MRA performed worse than DSA in identifying the arterial side of fistula anatomy. ${ }^{9}$

Finally, CE TR-MRA is a potentially attractive technique for DAVF follow-up. Noguchi et al. evaluated 21 treated transverse-sigmoid DAVFs with both DSA and CE TR-MRA to evaluate for both residual fistulas as well as retrograde venous drainage. They found that CE TRMRA identified the residual fistula in 8 of 9 cases and retrograde venous drainage in all 5 cases when compared to the gold-standard DSA. ${ }^{22}$ This accuracy in detecting retrograde drainage is particularly important in cases in which embolization eliminates the malignant drainage pattern but a small fistula still remains, as it allows for easy, regular-interval follow-up to ensure that the drainage pattern does not shift to a more malignant variety. Figure 2 demonstrates an example of following a DAVF with CE TR-MRA.

In our practice, CE TR-MRA has become the study of choice for patients with suspected cDAVFs, such as patients presenting with pulsatile tinnitus. After treatment, our center uses DSA to evaluate for cure either after embolization or after surgical ligation. In patients with DAVFs 

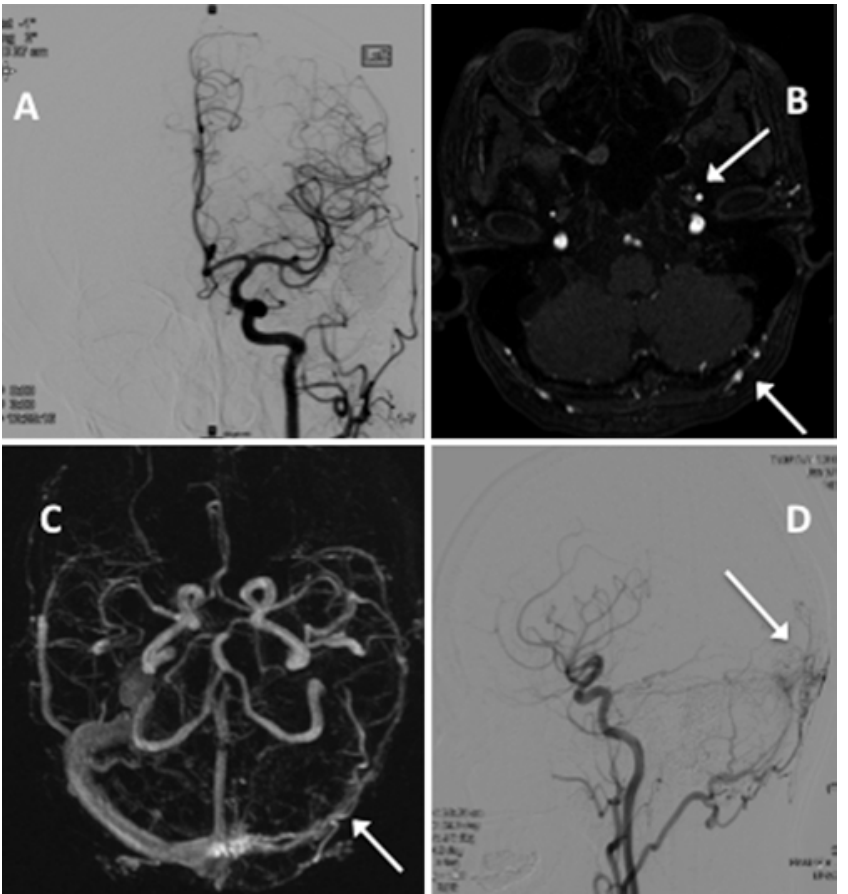

FIG. 1. Images obtained in a middle-aged woman who had previously been treated twice for Borden type 2 and type 3 transverse and sigmoid sinus DAVFs on the left. A cerebral angiogram performed for ear pain demonstrated complete angiographic cure (A). The patient developed new tinnitus a year later. TOF MRA demonstrated dilated external carotid artery branches (arrows) suspicious for a recurrent fistula (B). Static single frame from maximum intensity projection (MIP) cine CE TR-MRA (C) and then DSA (D) demonstrated recurrence of a Borden type 1 DAVF (arrows) supplied by the bilateral occipital arteries, bilateral meningohypophyseal trunks, and left posterior meningeal artery with drainage into the torcula.

without retrograde drainage, we use CE TR-MRA to follow these patients to ensure that there is no change in their drainage pattern.

\section{Cerebral Aneurysms}

The final intracranial vascular application of CE TRMRA is in the evaluation of intracranial aneurysms. While the literature is full of studies looking at the use of TOF MRA and CE MRA in the evaluation of new and coil-embolized aneurysms, CE TR-MRA has some specific uses for certain types of endovascularly treated aneurysms ${ }^{18,26}$ In these cases, the use of adjunctive laser-cut stents causes significant susceptibility artifact or radiofrequency shielding in other MRA techniques. Choi et al. analyzed 26 patients with machine-cut stent-assisted coil embolization and found superior visualization of the stented arteries with CE TR-MRA compared with TOF MRA. Their study also compared MRA techniques to DSA and found that CE TR-MRA was superior in evaluating the degree of aneurysm embolization compared to TOF MRA. ${ }^{5}$

Similar to machine-cut stents, braided flow diverters present a challenge for traditional MRA techniques due to the high metal surface coverage of the artery causing radiofrequency shielding. Our center published a study of
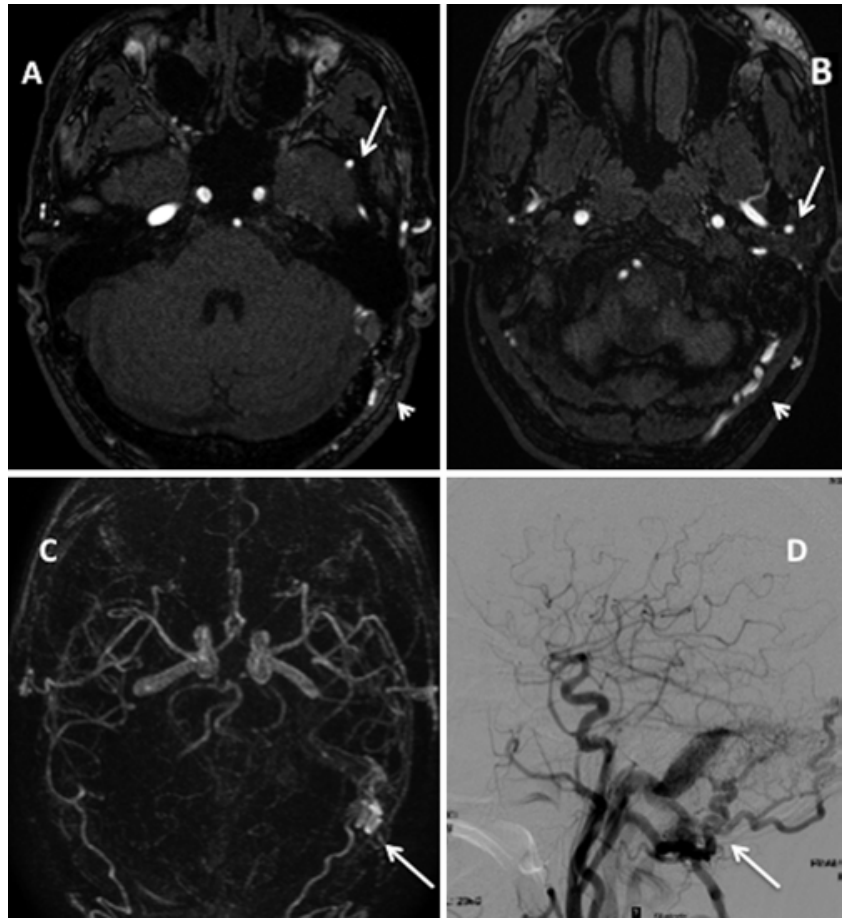

FIG. 2. Images obtained from a middle-aged man presenting to his primary care doctor with pulsatile tinnitus and negative CTA. TOF MRA demonstrated enlarged external carotid branches (arrows) and increased vascularity (arrowheads) along the transverse sinus (A and B). Static single frame from MIP cine CE TR-MRA demonstrated a Borden type I DAVF (arrow) fed by the left middle meningeal and occipital arteries with drainage into the transverse sinus (C). This was confirmed on DSA (arrow) and treated conservatively due to a lack of cortical reflux (D).

37 flow diverter-treated aneurysms and found both positive and negative predictive values (PPVs and NPVs) of $92 \%$ when comparing CE TR-MRA to DSA in terms of aneurysm occlusion. Visualization of the parent artery and measurement of luminal diameter was far superior with CE TR-MRA compared to TOF MRA. ${ }^{3}$ The results of this study shifted our long-term follow-up of flow-diverted aneurysms from DSA to CE TR-MRA. Figure 3 shows an example of a flow diverter-treated aneurysm followed on CE TR-MRA.

The one major limitation of this technique is potential susceptibility artifact in patients who have undergone surgical clip placement of nearby aneurysms, and alternative imaging modalities should be pursued in those situations (Table 1). In our practice, CE TR-MRA has become the study of choice for long-term follow-up of endovascularly treated aneurysms, especially those treated with flow diversion. We often will repeat DSA at 6 months, and if there are no issues we will then switch to CE TR-MRA to follow treated aneurysms, both those with complete occlusion and those with remnants.

\section{Spinal DAVFs}

The final vascular application of CE TR-MRA is in diagnosis of the rare entity of spinal DAVFs (sDAVFs). 


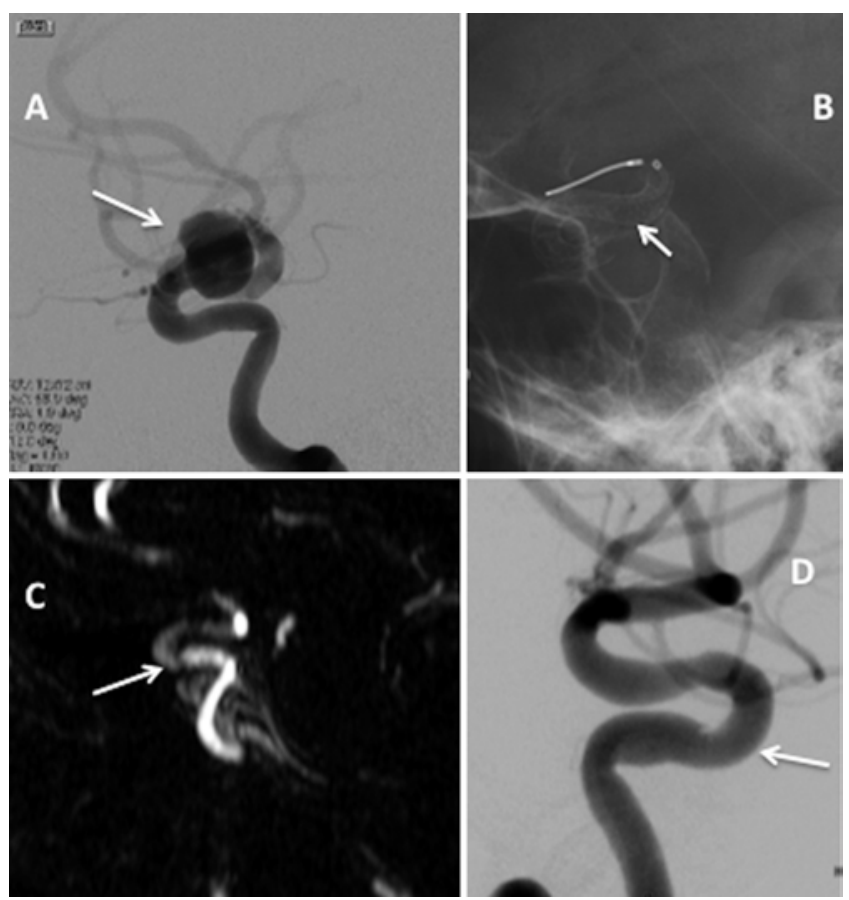

FIG. 3. Images from a middle-aged woman who presented with vision loss and was found to have a large ophthalmic-segment carotid artery aneurysm (arrow) on DSA (A). This was treated with a flow diverter (arrow; B). Six-month follow-up CE TR-MRA demonstrated complete aneurysm occlusion with mild in-stent stenosis (arrow; C). These findings (arrow) were confirmed on DSA (D).

These lesions consist of an abnormal connection between a radiculomeningeal artery and a radicular vein, most commonly at the level of the nerve root sleeve. This abnormal shunting leads to increased spinal venous pressure and progressive myelopathy. ${ }^{15}$ While the presence of these lesions can be suggested with high confidence on MRI due to associated abnormal flow voids and spinal cord edema, detection of the exact site of the fistulous connection is far more challenging noninvasively. On DSA this is often a long and difficult procedure as the neurointerventionalist needs to interrogate all vessels that can feed the spinal cord from the cervical carotids to the iliac arteries. Ac- cordingly, MRA has become a helpful adjunct to guide the neurointerventionalist to the level of interest, which accordingly shortens procedure time and limits iodinated contrast usage and radiation exposure.

To evaluate the accuracy of CE TR-MRA, Amarouche et al. evaluated 55 patients with suspected spinal AVMs or sDAVFs and found a PPV and NPV of $93 \%$ and $83 \%$, respectively. In the sDAVF group, CE TR-MRA accurately predicted the level of the fistula within 2 levels in $30(91 \%)$ of 33 cases and the correct side in $22(67 \%)$ of 33 cases. $^{2}$ Similarly, our group studied the Emory experience with 18 patients suspected of having an sDAVF and found a PPV and NPV of $88 \%$ and $90 \%$, respectively. In 6 of the 7 cases correctly diagnosed on CE TR-MRA, MRA predicted the fistula within 1 vertebral level. As an expected corollary, patients with negative or suboptimal CE TR-MRA, who then underwent DSA, had a significantly increased number of levels catheterized and volume of contrast used..$^{25}$ This study has changed our institution's clinical practice to prescreen all suspected sDAVFs with CE TR-MRA prior to DSA in the hopes of shortening the procedure. In addition to these 2 larger studies, multiple small studies demonstrate similar accuracy of CE TR-MRA when compared to DSA. 1,16,19,24 An example of CE TR-MRA used in the diagnosis of an sDAVF is demonstrated in Fig. 4.

Similarly to our center's paper mentioned above, ${ }^{25}$ we use CE TR-MRA to evaluate all potential sDAVFs prior to DSA. If the CE TR-MRA is negative, then we perform a full cranial-spinal DSA. If the CE TR-MRA has a potential culprit level, then we begin our DSA at that area of the spine and continue to the entire DSA if nothing is found. We continue to confirm obliteration with postembolization or postsurgical DSA. If there is any concern for incomplete treatment or recurrence, then our first test is CE TR-MRA.

\section{Conclusions}

CE TR-MRA, with its ability to hemodynamically assess lesion vascularity and venous drainage patterns, has a number of usages in cerebrovascular pathology. As experience with this technology and the technology itself improves, it will continue to play a larger role in the screening, evaluation, and follow-up of patients and allow many patients to avoid invasive DSA.

TABLE 1. Advantages and disadvantages of different imaging modalities

\begin{tabular}{lcc}
\hline Modality & \multicolumn{1}{c}{ Advantages } & Disadvantages \\
\hline TOF MRA & $\begin{array}{c}\text { Does not require iodinated or gadolinium contrast; facile } \\
\text { \& standardized post-processing; no ionizing radiation }\end{array}$ & $\begin{array}{c}\text { Degraded by treatment-related susceptibility artifact; } \\
\text { static flow-related imaging }\end{array}$ \\
\hline CE TR-MRA & $\begin{array}{c}\text { Dynamic evaluation of vascular enhancement during } \\
\text { contrast administration; can be optimized to reduce } \\
\text { treatment-related susceptibility artifact; no ionizing } \\
\text { radiation }\end{array}$ & $\begin{array}{c}\text { Requires gadolinium contrast; more time-consuming } \\
\text { \& reader experience-dependent to interpret; lower } \\
\text { spatial \& temporal resolution than DSA }\end{array}$ \\
& $\begin{array}{c}\text { Ubiquitously available; rapid scanning; high accuracy in } \\
\text { nontreated patients }\end{array}$ & $\begin{array}{c}\text { Degraded by treatment-related streak artifact; requires } \\
\text { iodinated contrast; ionizing radiation }\end{array}$ \\
\hline CTA & $\begin{array}{c}\text { Gold standard for vascular imaging; high spatial resolu- } \\
\text { tion; gives real-time flow-related information }\end{array}$ & $\begin{array}{c}\text { Invasive; costly; requires iodinated contrast; ionizing } \\
\text { radiation }\end{array}$ \\
\hline
\end{tabular}



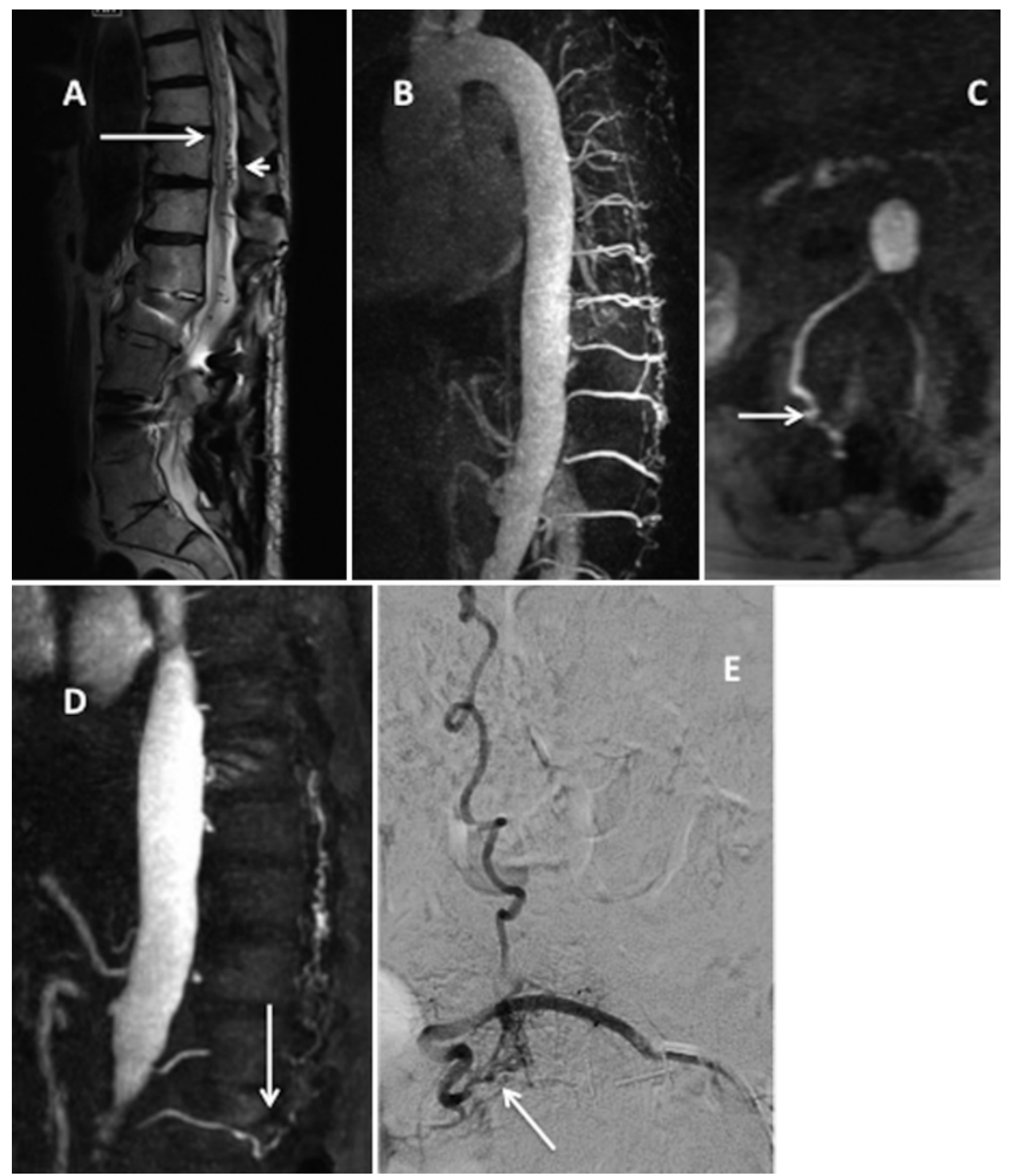

FIG. 4. Images from an elderly man who presented with a multiple-month history of lower-extremity weakness and numbness. He had a history of multiple lumbar surgeries in the past with significant blood loss. MRI of his thoracolumbar spine demonstrated cord signal change (arrow) with some prominent flow voids (arrowhead; A). CE TR-MRA demonstrated a likely sDAVF (arrow) from the right L2 radicular artery (B-D). DSA demonstrated a right L2 SDAVF (arrow) for which the patient underwent surgical ligation (E).

\section{References}

1. Ali S, Cashen TA, Carroll TJ, McComb E, Muzaffar M, Shaibani A, et al: Time-resolved spinal MR angiography: initial clinical experience in the evaluation of spinal arteriovenous shunts. AJNR Am J Neuroradiol 28:1806-1810, 2007

2. Amarouche M, Hart JL, Siddiqui A, Hampton T, Walsh DC: Time-resolved contrast-enhanced MR angiography of spinal vascular malformations. AJNR Am J Neuroradiol 36:417422, 2015

3. Boddu SR, Tong FC, Dehkharghani S, Dion JE, Saindane AM: Contrast-enhanced time-resolved MRA for follow-up of intracranial aneurysms treated with the pipeline embolization device. AJNR Am J Neuroradiol 35:2112-2118, 2014

4. Borden JA, Wu JK, Shucart WA: A proposed classification for spinal and cranial dural arteriovenous fistulous mal- formations and implications for treatment. J Neurosurg 82:166-179, 1995

5. Choi JW, Roh HG, Moon WJ, Kim NR, Moon SG, Kang CH, et al: Time-resolved 3D contrast-enhanced MRA on 3.0T: a non-invasive follow-up technique after stent-assisted coil embolization of the intracranial aneurysm. Korean J Radiol 12:662-670, 2011

6. Cognard C, Gobin YP, Pierot L, Bailly AL, Houdart E, Casasco A, et al: Cerebral dural arteriovenous fistulas: clinical and angiographic correlation with a revised classification of venous drainage. Radiology 194:671-680, 1995

7. Cuong NN, Luu VD, Tuan TA, Linh LT, Hung KD, Ngoc VTN, et al: Conventional digital subtractional vs noninvasive MR angiography in the assessment of brain arteriovenous malformation. Clin Neurol Neurosurg 169:29-33, 2018 
8. da Costa L, Wallace MC, Ter Brugge KG, O'Kelly C, Willinsky RA, Tymianski M: The natural history and predictive features of hemorrhage from brain arteriovenous malformations. Stroke 40:100-105, 2009

9. Ertl L, Brückmann H, Kunz M, Patzig M, Brem C, Forbrig R, et al: Assessment and treatment planning of lateral intracranial dural arteriovenous fistulas in 3 T MRI and DSA: a detailed analysis under consideration of time-resolved imaging of contrast kinetics (TRICKS) and ce-MRA sequences. Eur Radiol 26:4284-4292, 2016

10. Farb RI, Agid R, Willinsky RA, Johnstone DM, Terbrugge KG: Cranial dural arteriovenous fistula: diagnosis and classification with time-resolved MR angiography at 3T. AJNR Am J Neuroradiol 30:1546-1551, 2009

11. Frayne R, Grist TM, Korosec FR, Willig DS, Swan JS, Turski PA, et al: MR angiography with three-dimensional MR digital subtraction angiography. Top Magn Reson Imaging 8:366-388, 1996

12. Grist TM, Mistretta CA, Strother CM, Turski PA: Time-resolved angiography: past, present, and future. J Magn Reson Imaging 36:1273-1286, 2012

13. Hadizadeh DR, Kukuk GM, Steck DT, Gieseke J, Urbach H, Tschampa HJ, et al: Noninvasive evaluation of cerebral arteriovenous malformations by $4 \mathrm{D}-\mathrm{MRA}$ for preoperative planning and postoperative follow-up in 56 patients: comparison with DSA and intraoperative findings. AJNR Am J Neuroradiol 33:1095-1101, 2012

14. Hadizadeh DR, von Falkenhausen M, Gieseke J, Meyer B, Urbach H, Hoogeveen R, et al: Cerebral arteriovenous malformation: Spetzler-Martin classification at subsecondtemporal-resolution four-dimensional MR angiography compared with that at DSA. Radiology 246:205-213, 2008

15. Hurst RW, Kenyon LC, Lavi E, Raps EC, Marcotte P: Spinal dural arteriovenous fistula: the pathology of venous hypertensive myelopathy. Neurology 45:1309-1313, 1995

16. Jablawi F, Nikoubashman O, Schubert GA, Dafotakis M, Hans FJ, Mull M: Clinical and radiologic characteristics of deep lumbosacral dural arteriovenous fistulas. AJNR Am J Neuroradiol 39:392-398, 2018

17. Machet A, Portefaix C, Kadziolka K, Robin G, Lanoix O, Pierot L: Brain arteriovenous malformation diagnosis: value of time-resolved contrast-enhanced MR angiography at 3.0T compared to DSA. Neuroradiology 54:1099-1108, 2012

18. Majoie CB, Sprengers ME, van Rooij WJ, Lavini C, Sluzewski M, van Rijn JC, et al: MR angiography at 3T versus digital subtraction angiography in the follow-up of intracranial aneurysms treated with detachable coils. AJNR Am J Neuroradiol 26:1349-1356, 2005

19. Mathur S, Symons SP, Huynh TJ, Muthusami P, Montanera W, Bharatha A: First-pass contrast-enhanced MRA for pretherapeutic diagnosis of spinal epidural arteriovenous fistulas with intradural venous reflux. AJNR Am J Neuroradiol 38:195-199, 2017

20. Meckel S, Maier M, Ruiz DS, Yilmaz H, Scheffler K, Radue EW, et al: MR angiography of dural arteriovenous fistulas: diagnosis and follow-up after treatment using a time-resolved 3D contrast-enhanced technique. AJNR Am J Neuroradiol 28:877-884, 2007
21. Mohr JP, Parides MK, Stapf C, Moquete E, Moy CS, Overbey JR, et al: Medical management with or without interventional therapy for unruptured brain arteriovenous malformations (ARUBA): a multicentre, non-blinded, randomised trial. Lancet 383:614-621, 2014

22. Noguchi K, Kuwayama N, Kubo M, Kamisaki Y, Tomizawa G, Kameda K, et al: Dural arteriovenous fistula involving the transverse sigmoid sinus after treatment: assessment with magnetic resonance digital subtraction angiography. Neuroradiology 49:639-643, 2007

23. Oleaga L, Dalal SS, Weigele JB, Hurst RW, Lee J, Voorhees A, et al: The role of time-resolved 3D contrast-enhanced MR angiography in the assessment and grading of cerebral arteriovenous malformations. Eur J Radiol 74:e117-e121, 2010

24. Riccioli LA, Marliani AF, Ghedin P, Agati R, Leonardi M: CE-MR angiography at 3.0 T magnetic field in the study of spinal dural arteriovenous fistula. Preliminary results. Interv Neuroradiol 13:13-18, 2007

25. Saindane AM, Boddu SR, Tong FC, Dehkharghani S, Dion JE: Contrast-enhanced time-resolved MRA for pre-angiographic evaluation of suspected spinal dural arterial venous fistulas. J Neurointerv Surg 7:135-140, 2015

26. Schaafsma JD, Velthuis BK, Majoie CB, van den Berg R, Brouwer PA, Barkhof F, et al: Intracranial aneurysms treated with coil placement: test characteristics of follow-up MR angiography-multicenter study. Radiology 256:209-218, 2010

27. Soize S, Bouquigny F, Kadziolka K, Portefaix C, Pierot L: Value of 4D MR angiography at 3T compared with DSA for the follow-up of treated brain arteriovenous malformation. AJNR Am J Neuroradiol 35:1903-1909, 2014

28. Spetzler RF, Martin NA: A proposed grading system for arteriovenous malformations. J Neurosurg 65:476-483, 1986

\section{Disclosures}

Dr. Grossberg reports being a consultant to Cognition Medical, and having direct stock ownership in Cognition Medical and NTI.

\section{Author Contributions}

Conception and design: all authors. Drafting the article: Grossberg, Howard. Critically revising the article: all authors. Reviewed submitted version of manuscript: all authors. Approved the final version of the manuscript on behalf of all authors: Grossberg. Study supervision: Saindane.

\section{Correspondence}

Jonathan A. Grossberg: Emory University School of Medicine, Atlanta, GA. jonathan.a.grossberg@emory.edu. 